\title{
Can re-cTURBT be useful in pT1HG disease as a risk indicator of recurrence and progression? A single centre experience
}

\author{
Roberto Giulianelli ${ }^{1}$, Barbara Cristina Gentile ${ }^{1}$, Gabriella Mirabile ${ }^{1}$, Luca Albanesi ${ }^{1}$, Paola Tariciotti ${ }^{1}$, \\ Giorgio Rizzo $^{1}$, Maurizio Buscarini ${ }^{2}$, Mauro Vermiglio ${ }^{3}$ \\ ${ }^{1}$ CUrA, Nuova Villa Claudia Clinic, Rome, Italy; \\ ${ }^{2}$ Campus Biomedico, Rome, Italy; \\ ${ }^{3}$ Villa Gioia Clinic, Sora (FR), Italy.
}

\begin{abstract}
Summary Introduction: Understaging after initial transurethral resection is common in patients with high-risk non muscle infiltrating bladder cancer (NMIBC) and can delay accurate diagnosis and definitive treatment. The rate of upstaging from $T 1$ to $T 2$ disease after repeated transurethral resection ranges from 0 to $28 \%$, although the rate of upstaging may be even higher up to $49 \%$ when muscularis propria is absent in the first specimen. A restaging classic transurethral resection of bladder tumour (re-cTURBT) is the better predictor of early stage progression. According to some reports, the rate of positivity for tumor in re-cTURBT performed within eight weeks after initial cTURBT was as high as $18-77 \%$, and in about $40 \%$ of the patients a change in tumor stage was reported. We aimed to investigate, in high risk group, the presence of residual tumor following white light classical transurethral resection of bladder tumor (WLre-cTURBT) and the different recurrence and progression rate between patients with persistent or negative (pT0) onco-
\end{abstract} logical disease after WLre-cTURBT.

Materials and methods: A cohort of 285 patients presenting with primitive bladder cancer underwent to WLCTURBT from January 2011 to December 2015; out of them 92 (32.28\%) were T1HG. In according to EAU guidelines 2011, after 4-6 weeks all HG bladder cancer patients underwent a WL recTURBT . All patients were submitted to a subsequent followup including cystoscopy every 3 months with multiple biopsies, randomly and in the previous zone of resection; urinary citology on 3 specimens and kidney/bladder ultrasound every 6 months. The average follow-up was 48 months.

Results: Following WLre-cTURBT we observed a persistent disease in 18 (15.2\%) patients: 14 (77.7\%) with a HG-NMIBC and $4(22.2 \%)$ with a high grade (HG) muscle invasive bladder cancer (pT2HG). After follow up of all 92 patients according to the guidelines EAU, we observed recurrence in 36/92 (39.1\%) and progression in 14/92 (15.2\%). Of 14 NMIBC with persistent disease, 10 patients $(71.4 \%)$ showed recurrence: 4 patients (40\%) were pT1HG with concomitant carcinoma in situ (CIS), 3 patients (30\%) multifocal pTaHG, 2 (20\%) patients CIS and one patient (10\%) a muscle invasive neoplasm (pT2HG). Instead of the group of 48 patients pT0 following WL recTURBT, we observed recurrence in 26 patients (54.1\%) and in two patients $(4.1 \%)$ progressions, who presented after 3 months in association with CIS. The remaining 22 patients (45.9\%) with initial pT1HG are still progression free.

Multivariate analysis showed that the most important variable of early progression were persistent neoplasm and histopathological findings at WLre-cTURBt $(p=0.01)$, followed by the result of the first cystoscopy $(p=0.002)$ and presence of CIS $(p=0.02)$.

Discussion: Following WLre-cTURBt in HG-NMIBC patients we identified in $15 \%$ of cases a persistent disease with a $4.3 \%$ of MIBC. In the high risk persistent bladder neoplasms group we observed recurrent and progression rate higher than in TO bladder tumours group $(\Delta=+17.3 \%$ and $\Delta=+62.5 \%$, $p<0.05)$.

KEY WORDS: pT1HG; WLre-cTURBT; BCG schedule; pCISHG Recurrence and progression rate.

Submitted 14 November 2016; Accepted 11 January 2017

\section{INTRODUCTION}

Bladder cancer is a common genito-urinary malignancy, with transitional cell carcinoma comprising nearly $90 \%$ of all primary bladder tumours. At the first diagnosis $70 \%$ to $80 \%$ of urothelial tumours are confined to the epithelium, the remainder is characterized by muscle invasion. A significant number of patients with high risk non-muscle invasive bladder tumours (HG-NMIBT) treated with white light classic transurethral resection of bladder tumours (WLCTURBT) and intravesical BCG will progress to invasive disease (1-3). Progression to muscle invasion (pT2) mandates immediate radical cystectomy (4).

WLcTURBT is the standard initial therapy for NMIBT, but the high percentage of recurrence after surgery is still an unresolved problem (5). High grade pT1 bladder neoplasm (pT1HG) really represents a therapeutic challenge due to the high risk of progression (about 15-30\%) to muscle-invasive disease, usually within 5 years (6).

However, no consensus exists regarding the treatment of patients with recurrent bladder tumours that invade the lamina propria (pT1) (7-9). Recent studies suggested that the first cTURBT may be incomplete in a significant number of cases (10). Understaging at the time of the initial transurethral resection is common for patients with high-risk NMIBC and can delay accurate diagnosis and definitive treatment. It is therefore recommended for patients with high-risk disease and in those with large or multiple tumors or when the initial transurethral resection is incomplete, to repeat WLre-cTURBT within 2-6 
weeks in order to guide appropriate management (11). We aimed to investigate, in high risk group, the presence of residual tumor following WLcTURBT and the different recurrence and progression rate between patients with persistent or negative (pT0) oncological disease after WLre-cTURBT.

\section{Materials AND Methods}

In our department from January 2011 to June 2015, 285 consecutive patients with a first diagnosis of clinical bladder tumor underwent WLcTURBT.

Histopathological findings showed 95 high grade (HG) bladder tumours invading the lamina propria ( $\mathrm{pTl}$ ) but only 92 (32.28\%) cases were eligible for our study.

Patients mean age was 68 years (range 42 to 78 yrs, SD 10.4); 22 patients were women; mean follow-up 28 months (range 16-5 months). Focality and dimensions are reported Table 1 . We performed WLcTURBT with complete resection of all visible lesions and tumor bed and margins were taken separately. All resections were performed in our institution by the same experienced surgeon, R.G.

Our exclusion criteria included patients that underwent an incomplete resection or cases whose specimens were without muscle tissue in order to evaluate tumour invasion. We excluded three patients: two because of the lack of muscle tissue in the specimen and one because of the incomplete resection. A WLre-cTURBT, according to EAU guidelines (11), was routinely performed within 4 to 6 weeks following the first resection if the histopathological findings revealed Tl tumour. The surgeon performed the procedure with the same technique of the initial WLCTURBT: complete resection of all suspect residual tumour, separate resection of the underlying bladder wall with an adequate amount of detrusor muscle, wide resection of the margins to exclude the presence of carcinoma in situ (CIS) and associated biopsies with a loop resection ('selected biopsies') of the abnormal areas of urothelium. If there was not residual tumour or if it was superficial, intravesical immunotherapy conforming to Lamm's schedule was planned (12). In according to EAU guidelines (11), urine cytology and follow-up cystoscopy were performed every 3-month for the first year, biannually for the second year and annually thereafter. Ultrasonography of the urinary upper tract was performed every six months. However, if muscle invasive residual tumour or CIS were detected, subsequent treatment strategy was radical cystoprostatectomy.

Recurrence Free Survival, Progression Free Survival and Overall Survival curves were calculated by the KaplanMeier method (13) and compared by the log rank test (14). Statistical analysis was performed using the Pearson chi-square test (15). Tumors were classified according to the TNM system of the UICC (16) and Grading according to WHO classification. Patients were fully informed and consented the procedure.

\section{RESULTS}

Of the 95 enrolled patients, 92 were considered evaluable for the actual analysis. After WLre-cTURBT performed within 4 to 6 weeks following the first resection, we observed 18 (19.5\%) patients with recurrence: 4 (4.34\%) with muscle invasive bladder cancer (MIBC) and 14 (15.2\%) with NMIBC (Table 2). Regarding focality, dimension and concomitant CIS, all patients with invasive neoplasms were multifocal $(p<0.01),>3 \mathrm{~cm}(\mathrm{p}<0.01)$ or with concomitant CIS ( $\mathrm{p}<0.001)$ to the first WLcTURBT.

All MIBC patients underwent radical cystoprostatectomy with staging lymphadenectomy and the histopathological evaluation revealed one patient with no evidence of TCC in the cystectomy specimen (pT0) and three patients with muscle invasion (pT2b) but no evidence of positive lymphnodes ( $\mathrm{pNO}$ ).

We carried out the follow up of all 92 patients according to the EAU guidelines, and we found out recurrence in $36 / 92(39.1 \%)$ and progression in 14/92 (15.2\%).

All the 14 patients (15.2\%) with NMIBC underwent intravesical immunotherapy conforming to Lamm's schedule (12) and $10(71.4 \%)$ of them showed recurrences respectively after $4,6,7,10$ and 12 months (see Table 3). Of these 10 patients, 4 patients (40\%) showed recurrences pTlHG with concomitant CIS, 3 patients $(30.0 \%)$ multifocal pTaHG, two (20.0\%) CIS and one (10.0\%) a muscle invasive neoplasm (pT2HG). All these patients with high grade persistent disease following BCG-schedule were regarded as "patients progressing" and after pelvis-abdomen CT and scintigraphy, radical cystoprostatectomy with staging lymphadenectomy was suggested.

The histopathological evaluation revealed two patients $(2.17 \%)$ with no evidence of TCC in the cystectomy specimen (pT0), six patients (6.52\%) with muscle invasive tumor pT2a and two (2.17\%) pT2b, with no evidence of positive lynphnodes (pNO). All of the patients are actually living and disease-free but one (1.08) with muscle invasive tumor (pT2aNO) who died for another cause without evidence of disease progression. In the group of patients with

Table 1.

T1HG patients: focality and dimensions.

\begin{tabular}{|lccc|}
\hline Focality & Pts & Dimensions & Pts \\
\hline Unifocal & 14 & $<1 \mathrm{~cm}$ & 12 \\
\hline Multifocal & 78 & $>1<3 \mathrm{~cm}$ & 54 \\
\hline & 92 & $>3 \mathrm{~cm}$ & 26 \\
\hline & & 92 \\
\hline
\end{tabular}

Table 2.

TIHG patients reTURB: recurrence and progression.

\begin{tabular}{|lccc|}
\hline Re-TURB & Pts & pT1 & pT2 \\
\hline Recurrences & & & Progression \\
\hline$\%$ & 18 & 14 & 4 \\
\hline
\end{tabular}

Table 3.

TaHG patients follow-up: BCG, recurrence and progression.

\begin{tabular}{|lcccccc|}
\hline BCG & Rec & $4 \mathrm{mo}$ & $6 \mathrm{mo}$ & $7 \mathrm{mo}$ & $10 \mathrm{mo}$ & $12 \mathrm{mo}$ \\
\hline 14 & & $\mathrm{pT} 1 \mathrm{HG} / \mathrm{ClS}$ & $\mathrm{pTaHG}$ & $\mathrm{CIS}$ & $\mathrm{pT} 2 \mathrm{HG}$ & $\mathrm{pT} 1 \mathrm{HG} / \mathrm{ClS}$ \\
\hline $\mathrm{Pts}$ & 10 & 3 & 3 & 2 & 1 & 1 \\
\hline $15.20 \%$ & $10.87 \%$ & $3.27 \%$ & $3.27 \%$ & $2.17 \%$ & $1.08 \%$ & $1.08 \%$ \\
\hline
\end{tabular}


Figure 1.

Kaplan-Meier overall recurrence survival analysis.

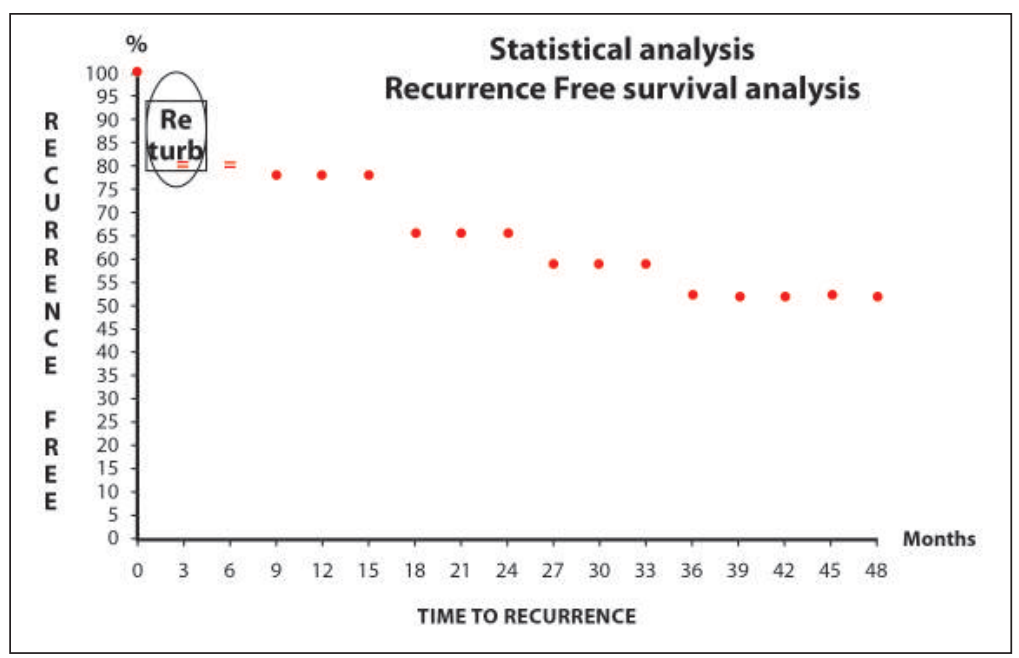

Figure 2.

Kaplan-Meier overall progression survival analysis.

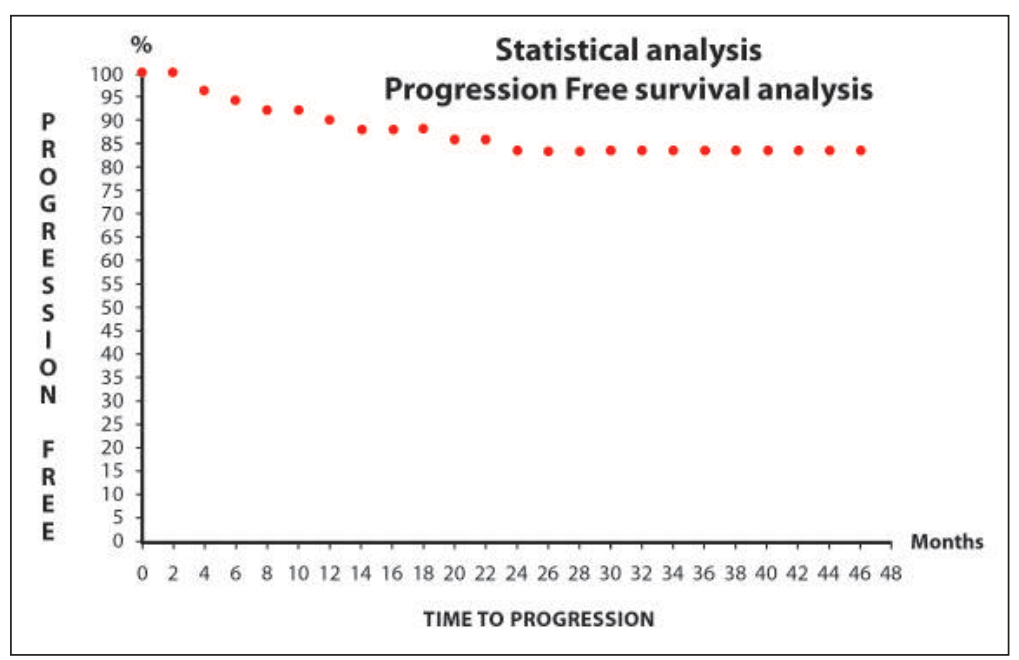

cTURBT is strongly recommended regardless of the presence of muscle in specimens because of the possibility of understating due to incomplete resection $(17,18)$. It provides more accurate pathological staging information, since persistent tumor in second cTURBT specimens can be detected in $33 \%-55 \%$ of patients $(19,20)$. In our experience we observed persistent bladder lesions after WLre-cTURBT in $19.5 \%$ of patients. In addition, a WLre-cTURBT promotes cancer control. In a randomized controlled study, re-cTURBT decreased the recurrence rate compared to a single cTURBT (21). In our expeience, in the persistent group, recurrences and progressions were more elevated than in TO group. Persistent disease after WLre-cTURBt is a poor prognostic indicator of recurrence and progression.

Dutta et al. (22) demonstrated that, if a WLrecTURBT is performed, the risk of upstaging is near $30 \%$, but the risk of residual tumour is still significant. We already reported our experience concerning the need of WLrecTURBT in patients with primary pTl TCC of the bladder. Residual tumour rate was 19.5\% in 18 patients who underwent WLre-cTURBT following primary diagnosis of $\mathrm{Tl}$ disease and overall recurrence was 39.1\%. Residual neoplasms were detected only in multifocal tumor, concomitant CIS and $>3 \mathrm{~cm}$ at first pT1HG bladder tumours. Our opinion is that the main and most important rule is a complete resection of the NMIBC.

This procedure is not only mandatory for an adequate staging but also useful to the completion of WLCTURBT for most of the non muscle invasion tumours. An inadequate and incomplete resection increases recurrence rate as Brausi et al. (10) already showed: in

persistent disease following WLREcTURBT we observed a overall MIBC rate of $66.6 \%$ (12 pts). Instead of the group of 48 patients who were T0 following WLre-cTURBT and had intravesical immunotherapy (BCG) conforming to Lamm's schedule (12), we observed recurrence in 26 pts (54.1\%) and in only two patients (4.1\%) progression, who presented after 3 months, associated with CIS.

The remaining 22 patients (45.9\%) with initial T1HG are still progression free.

Figures 1 and 2 show Kaplan-Meier overall recurrencefree and progression-free curves, respectively.

\section{Discussion}

WLCTURBT is the main-stay approach in the diagnosis and treatment of bladder cancer. On the basis of the EORTC risk tables and prognostic factors for T1HG bladder tumours, the probability of recurrence at 1 and 5 year, respectively, is $24-61 \%$ and $46-78 \%$, and the probability of progression, respectively, is $1-17 \%$ and $6-45 \%$ (3). If patients are diagnosed with high-grade $\mathrm{Tl}$, a re- fact the curative effect of an excellent resection is especially showed in superficial disease. Grimm et al. (23) investigated the role of WLre-cTURBT in an heterogeneous group of patients with superficial bladder cancer. They found that the estimated risk of recurrence after 1, 2 and 3 years was 18\%, 29\% and 32\% respectively and recurrence was observed in 38\% pts treated with re-TURBT. Divrik et al. (24) found the recurrence rate was 13.6\%, $22.3 \%$ and $31.2 \%$ in the first, the second and the third year respectively and that overall recurrence was $25.6 \%$. Klan et al. (25) reported a residual tumour rate of $50 \%$ in patients with pT1 HG tumours; Herr (26) reported a rate of $74 \%$ residual tumours in 58 patients with pTl HG bladder cancer, while Mersdorf et al. (27) detected residual tumours in 58\% (26 of 45 patients) pT1HG bladder cancer. WLre-cTURBT certainly detected a significant percentage of residual tumours and, among them, CIS and muscle invasive disease rates were reported with a range of $6 \%$ to $24 \%$ in different studies $(28,29)$.

Kitamura and Kakehi (30) suggested that optimal management strategies should be based on pathological find- 
ings from second cTURBT specimens in patients with T1 disease. They recommended that patients with T0 upon WLre-cTURBT should be considered for BCG therapy or watchful waiting. A randomized controlled study is ongoing comparing watchful waiting to BCG therapy in high-grade T1 disease with T0 on WLre-cTURBT.

BCG instillation into bladder is the gold standard for conservative treatment for high-grade $\mathrm{Tl}$ disease. The therapeutic effect of BCG in high-grade T1 has already been established by several meta-analysis studies $(31,32)$. However, we have to keep in mind that patients die upon progression to MIBC, not upon recurrence, and that the effectiveness of BCG at preventing progression was not as great as its effectiveness at preventing recurrence. Further, residual tumor in the WLre-cTURBT specimen is associated with poor prognosis. In our experience, we observed progressions in 14/92 patients (15.2\%): 12 from the group with persistent disease group and two in T0 patients. In the persistent disease group, we identified four patients with muscle inasive disease at WLrecTURBT and 8 more patients during the follow-up. According to EAU guidelines (11), all patients were submitted to radical cystectomy and staging lymphadenectomy. All patients who had a muscle-invasive disease, confined within the muscle layer, without extra-parietal extension or lymphadenopathy, are today living (NED). Early cystectomy was mandatory to have good results. Cystectomy has definite advantages for high-grade Tl disease. In the largest study so far, the clinical outcomes of 167 patients with high-grade Tl were reviewed after cystectomy (33). Surprisingly, almost 30\% experienced disease recurrence, and $18.5 \%$ died from bladder cancer. Half of the cases had disease upstaging, and $27.5 \%$ had extravesical disease. A greater than 3-month delay between cystectomy and last WLCTURBT showed a trend toward upstaging, which means that delaying cystectomy for BCG therapy may worsen prognosis.

Herr et coll showed that of 92 patients with residual T1 cancer in WLre-cTURBT, 75 (82\%) progressed to muscle invasion within 5 years compared to 49 of 260 (19\%) who had no or non-Tl tumor detected on restaging cTURBT (34). A similar study reported that early cystectomy seems to prolong cancer-specific survival compared to deferred cystectomy in high-risk high-grade Tl patients (35).

Considering the high risk of progression and cancer death of high-grade Tl disease, cystectomy would be the best answer for treatment. However, there are disadvantages. First, cystectomy may be overtreatment for highgrade Tl disease. Since at least $50 \%$ of high-grade T1 patients are not upstaged upon cystectomy (36). Second, cystectomy deteriorates the quality of life. Finally, cystectomy is a highly complicated surgery in the urological field, and almost $30 \%-50 \%$ of patients experience perioperative or long-term complications (37).

In our experience, the most important predictive prognostic factor - in patients with PTlHG tumours - is the presence of concomitant CIS. Sylvester et al. observed in pTlHG patients without CIS a probability of progression around $10 \%$ at 1 year and $29 \%$ at 5 years. In pT1HG patients with CIS, the corresponding features are 29\% and $74 \%$, respectively (3).

\section{Conclusions}

WLre-cTURBT is a useful tool because in a percentage of the patients this additional surgery results in an improvement of treatment strategy. True recurrence rate can be better evaluated by this approach because residual tumors may be erroneously defined as recurrence. Our data showed that it is necessary to perform a WLrecTURBT in patients with newly diagnosed, high grade (HG), stage pTl bladder cancer for a 'real' staging and a complete resection (15\% persistent disease). In our experience, following WLre-cTURBt in HGNMIBC risk group patients we dentified a $15 \%$ persistent disease with a $4.3 \%$ of MIBC. In the patients with persistent bladder neoplasms we observed a overall recurrence and progression rate more elevated than in T0 bladder tumours group $(\Delta=+17.3 \%$ and $\Delta=+62.5 \%)$.

\section{REFERENCES}

1. Andius P, Holmang S. Bacillus Calmette-Guerin therapy in stage Ta-T1 bladder cancer: prognostic factors for time to recurrence and progression. BJU Int. 2004; 93:980

2. Herr HW, Klein EA, Rogatko A. Local BCG failures in superficial bladder cancer. A multivariate analysis of risk factors influencing survival. Eur Urol. 1991; 19:97

3. Sylvester RJ, van der Meijden AP, Oosterlinck W, et al. Predicting recurrence and progression in individual patients with stage Ta-T1 bladder cancer using EORTC risk table: a combined analysis of 2596 patients from seven EORTC trials. Eur Urol. 2006; 49:466.

4. Segal R, Yafi FA, Brimo F, et al. Prognostic factors and outcome in patients with $T 1$ high-grade bladder cancer: can we identify patients for early cystectomy? BJU Int. 2012; 109:1026-30.

5. Kim HS, Ku JH, Kim SJ, et al. Prognostic factors for recurrence and progression in Korean non-muscle-invasive bladder cancer patients: a retrospective, multi-institutional study. Yonsei Med J. 2016; 57:855-64.

6. Nepple KG, O'Donnell MA. The optimal management of T1 highgrade bladder cancer. Can Urol Assoc J. 2009; 3 (6 Suppl 4):S188S192.

7. Brake M, Loertzer H, Horsch R, Keller H. Recurrence and progression of stage T1, grade 3 transitional cell carcinoma of the bladder following intravesical immunotherapy with Bacillus CalmetteGuerin. J Urol. 2000; 163:40.

8. Bianco FJ Jr, Justa D, Grignon DJ, et al. Management of clinical $T 1$ bladder transitional cell carcinoma by radical cystectomy. Urol Oncol. 2004; 22;290.

9. Smaldone MC, Jacobs BL, Smaldone AM, Hrebinko RL. Longterm results of selective partial cystectomy for invasive urothelial bladder carcinoma. Urology. 2008; 72:613-6.

10. Brausi M, Collette L, Kurth K, et al. Variability in the recurrence rate at first follow-up cystoscopy after TUR in stage Ta-T1 transitional cell carcinoma of the bladder: a combined analysis of seven EORTC studies. Eur Urol. 2002; 41:543.

11. Babjuk M, Oosterlinck W, Sylvester R, European Association of Urology (EAU), et al. EAU guidelines on non-muscle-invasive urothelial carcinoma of the bladder, the 2011 update. Eur Urol. 2011; 59:997-1008

12. Lamm DL, Blumenstein BA, Crissman JD, et al. Maintenance bacillus Calmette-Guérin immunotherapy for recurrent $\mathrm{Ta}, \mathrm{T} 1$ and carcinoma in situ transitional cell carcinoma of the bladder: A ran- 
domized Southwest Oncology Group Study. J Urol. 2000; 163:11241129.

13. Kaplan EL, Meier P. Nonparametric estimation from incomplete observations. J Amer Statist Assn. 1958; 53:457-481.

14. Harrington D. Linear Rank Tests in Survival Analysis, in Encyclopedia of Biostatistics, Wiley Interscience, 2005.

15. Pearson K. On the criterion that a given system of deviations from the probable in the case of a correlated system of variables is such that it can be reasonably supposed to have arisen from random sampling. The London, Edinburgh, and Dublin Philosophical Magazine and Journal of Science - Series 5 1900; 50:157-175.

16. Cheng L, Montironi R, Davidson DD, Lopez-Beltran A. Staging and reporting of urothelial carcinoma of the urinary bladder. Modern Pathology. 2009; 22:S70-S95;

17. Ramírez-Backhaus M, Dominguez-Escrig J, Collado A, et al. Restaging transurethral resection of bladder tumor for high-risk stage Ta and T1 bladder cancer. Curr Urol Rep. 2012; 13:109-114.

18. Shim JS, Choi H, Noh TI, et al. The clinical significance of a second transurethral resection for $\mathrm{T} 1$ high-grade bladder cancer: Results of a prospective study. Korean J Urol. 2015; 56:429-434.

19. Jahnson S, Wiklund F, Duchek M, et al. Results of second-look resection after primary resection of $T 1$ tumour of the urinary bladder. Scand J Urol Nephrol. 2005; 39:206-210.

20. Vasdev N, Dominguez-Escrig J, Paez E, et al. The impact of early re-resection in patients with pT1 high-grade non-muscle invasive bladder cancer. Ecancermedicalscience 2012; 6:269.

21. Kim W, Song C, Park S, et al. Value of immediate second resection of the tumor bed to improve the effectiveness of transurethral resection of bladder tumor. J Endourol. 2012; 26:1059-1064.

22. Dutta SC, Smith Jr JA, Shappell SB, et al. Clinical under staging of high risk nonmuscle invasive urothelial carcinoma treated with radical cystectomy. J Urol. 2004; 166:539.

23. Grimm MO, Steinhoff C, Simon X, et al. Effect of routine repeat transurethral resection for superficial bladder cancer a long-term observational study. J Urol 2003; 170:433.

24. Divrik RT, Yldirim U, Zorlu F, Ozen H. The effect of repeat transurethral resection on recurrence and progression rates in patients with T1 tumours of the bladder who received intravesical Mytomicin: A prospective, randomised clinical trial. J Urol. 2006; 175:1644.
25. Klan R, Loy V, Huland H. Residual tumour discovered in routine second transurethral resection in patients with stage T1 transitional cell carcinoma of the bladder. J Urol. 1991; 148:316.

26. Herr HW. The value of a second transurethral resection in evaluating patients with bladder tumours. J Urol. 1999; 162:74.

27. Mesdorf A, Brauers A, Wolff JM, et al. 2nd TUR for superficial bladder cancer, a must? J Urol. 1998; Suppl., 159:143, abstract 542.

28. Schips L, Augustin H, Zigeuner RE, et al. Is repeated transurethral resection justified in patients with newly diagnosed superficial bladder cancer? Urology. 2002; 60:822.

29. Ojea Calvo A, Nunuz Lopez A, Alonso Rodrigo A, et al. Value of a second transurethral resection in the assesment and treatment of patients with bladder tumour. Actas Urol Esp. 2001; 25:182.

30. Kitamura H, Kakehi Y. Treatment and management of highgrade T1 bladder cancer: what should we do after second TUR? Jpn J Clin Oncol. 2015; 45:315-322..

31. Shelley MD, Wilt TJ, Court J, et al. Intravesical bacillus Calmette-Guérin is superior to mitomycin $C$ in reducing tumour recurrence in high-risk superficial bladder cancer: a meta-analysis of randomized trials. BJU Int. 2004; 93:485-490.

32. Böhle A, Bock PR. Intravesical bacille Calmette-Guérin versus mitomycin $C$ in superficial bladder cancer: formal meta-analysis of comparative studies on tumor progression. Urology. 2004; 63:682686.

33. Gupta A, Lotan Y, Bastian PJ, et al. Outcomes of patients with clinical T1 grade 3 urothelial cell bladder carcinoma treated with radical cystectomy. Urology 2008; 71:302-307.

34. Herr HW, Donat SM, Dalbagni G. Can restaging transurethral resection of $\mathrm{T} 1$ bladder cancer select patients for immediate cystectomy? J Urol. 2007; 177:75-79.

35. Denzinger S, Fritsche HM, Otto W, et al. Early versus deferred cystectomy for initial high-risk pT1G3 urothelial carcinoma of the bladder: do risk factors define feasibility of bladder-sparing approach? Eur Urol. 2008; 53:146-152.

36. Lambert EH, Pierorazio PM, Olsson CA, et al. The increasing use of intravesical therapies for stage T1 bladder cancer coincides with decreasing survival after cystectomy. BJU Int. 2007; 100:33-36.

37. Hautmann RE, Abol-Enein H, Davidsson T, et al. ICUD-EAU International Consultation on Bladder Cancer 2012: Urinary diversion. Eur Urol. 2013; 63:67-80.

\section{Correspondence}

Roberto Giulianelli, MD

giulianelli0764@gmail.com

Barbara Cristina Gentile, MD

Gabriella Mirabile, MD

Luca Albanesi, MD

Paola Tariciotti, MD

paola.tariciotti@libero.it

Giorgio Rizzo, MD

CUrA, Nuova Villa Claudia Clinic, Rome, Italy

Maurizio Buscarini, MD

Campus Biomedico, Rome, Italy

Mauro Vermiglio, MD

Villa Gioia Clinic, Sora (FR), Italy 